ARTICLE

Received 14 Dec 2013 | Accepted 7 May 2014 | Published 4 Jun 2014 DOl: 10.1038/ncomms5057

\title{
Light-inducible receptor tyrosine kinases that regulate neurotrophin signalling
}

Ki-Young Chang ${ }^{1,2, \star}$, Doyeon Woo ${ }^{2,3, \star}$, Hyunjin Jung ${ }^{2}$, Sangkyu Lee ${ }^{1,2}$, Sungsoo Kim², Joungha Won², Taeyoon Kyung ${ }^{2}$, Hyerim Park ${ }^{2}$, Nury Kim²,2, Hee Won Yang ${ }^{2}$, Jae-Yong Park ${ }^{3,4}$, Eun Mi Hwang ${ }^{3}$, Daesoo $\mathrm{Kim}^{2} \&$ Won Do Heo ${ }^{1,2}$

Receptor tyrosine kinases (RTKs) are a family of cell-surface receptors that have a key role in regulating critical cellular processes. Here, to understand and precisely control RTK signalling, we report the development of a genetically encoded, photoactivatable Trk (tropomyosinrelated kinase) family of RTKs using a light-responsive module based on Arabidopsis thaliana cryptochrome 2. Blue-light stimulation $(488 \mathrm{~nm}$ ) of mammalian cells harbouring these receptors robustly upregulates canonical Trk signalling. A single light stimulus triggers transient signalling activation, which is reversibly tuned by repetitive delivery of blue-light pulses. In addition, the light-provoked process is induced in a spatially restricted and cell-specific manner. A prolonged patterned illumination causes sustained activation of extracellular signal-regulated kinase and promotes neurite outgrowth in a neuronal cell line, and induces filopodia formation in rat hippocampal neurons. These light-controllable receptors are expected to create experimental opportunities to spatiotemporally manipulate many biological processes both in vitro and in vivo.

\footnotetext{
${ }^{1}$ Center for Cognition and Sociality, Institute for Basic Science (IBS), Daejeon 305-701, Republic of Korea. ${ }^{2}$ Department of Biological Sciences, Korea Advanced Institute of Science and Technology (KAIST), Daejeon 305-701, Republic of Korea. ${ }^{3} \mathrm{WCI}$ Center for Functional Connectomics, Korea Institute of Science and Technology (KIST), Seoul 136-791, Republic of Korea. ${ }^{4}$ Department of Neurobiology, Institute of Health Science, and Medical Research Center for Neural Dysfunction, Gyeongsang National University School of Medicine, Jinju 660-751, Republic of Korea. * These authors contributed equally to this work. Correspondence and requests for materials should be addressed to W.D.H. (email: wondo@kaist.ac.kr).
} 
C ells respond to their environment by transducing external stimuli, for example growth factors and hormones, into relevant internal signals via cell-surface receptors, such as G-protein-coupled receptors ${ }^{1}$ and receptor tyrosine kinases $(\mathrm{RTKs})^{2}$. By binding to their ligands, receptors mediate the activation of diverse intracellular signalling pathways and regulate numerous cellular processes. Accordingly, natural ligands or synthetic agonists have been broadly used as indispensable tools to specifically control receptor activity and probe the mechanisms underlying cellular behaviours. However, they do not allow spatial control and have temporal resolution that is limited by time required for diffusion, making it difficult or impossible to dissect and analyse the dynamic nature of cellular signalling networks in a spatiotemporally precise manner. One promising approach to circumvent these limitations is coupling light signals to receptor activity. Because the activating stimulus (light) can be delivered with high spatiotemporal specificity, and even simply switched on and off, experimental opportunities are created that would not be possible with classical ligand-based and pharmacological methods. To date, efforts to control cell signalling through optical modulation of receptor activity have mainly used vertebrate rhodopsin, as exemplified by engineered chimeric receptors that combine rhodopsin with G-proteincoupled receptors ${ }^{3,4}$.

Recently developed light-responsive interacting modules ${ }^{5-7}$ may represent a new strategy for light activation of receptors. The members of a module pair, which are based on plant photoreceptors derived from Arabidopsis thaliana, are genetically encoded and allow control of protein-protein interactions in mammalian cells in a light-dependent fashion, thereby suitable for application to receptor systems that are activated by an interaction between receptor monomers, such as RTKs. Normally, RTK activation is induced by ligand-mediated receptor dimerization (Fig. 1a), which in turn leads to autophosphorylation of tyrosine kinase domains and recruitment of downstream signalling molecules ${ }^{2}$. However, this interaction, and thus receptor activation, can be induced artificially using chemical inducers of dimerization (CIDs) ${ }^{8}$. In this application, tyrosine kinase domains are fused to the binding domain(s) recognized by CIDs, allowing RTKs to be pharmacologically crosslinked and activated, independently of their endogenous counterpart and ligand, in the presence of CIDs such as rapamycin or AP20187 (refs 9-11). Accordingly, we reasoned that the lightmediated interactions could functionally substitute for the CID approach and allow RTKs to be dimerized and activated in response to light.
In this study, our goal was to develop photoactivatable RTKs. The tropomyosin-related kinase (Trk) family was chosen as a candidate light-induced RTK. Following binding to neurotrophins, the three members of this family, Trk A, B and C, mediate activation of intracellular signalling pathways, including mitogen-activated protein kinase kinase (MEK)/extracellular signal-regulated kinase (ERK), phosphatidylinositol 3-kinase (PI3K)/Akt and phospholipase C $\gamma 1$ (PLC $\gamma 1) / \mathrm{Ca}^{2+}$ pathways, and thereby contribute to neuronal survival, neurite outgrowth and synaptic plasticity ${ }^{12}$. As a light-inducible dimerization system, we initially introduced the photolyase homology region (PHR) of cryptochrome 2, a blue-light photoreceptor, and its optically driven interaction partner CIBN, an N-terminal domain of CIB1, because the PHR-CIBN pair achieves fast and precise control of protein-protein interactions upon blue-light exposure with no requirements for exogenous cofactors ${ }^{7}$. However, we unexpectedly found that PHR can be harnessed as a homointeraction domain to activate the linked Trk receptors in response to light (Fig. 1 and Supplementary Fig. 1). Exploiting this serendipitous outcome, we were able to achieve optically activatable Trk signalling using a single light-responsive module (Fig. 1b).

Here we show that light stimulation can activate canonical Trk signalling pathways through photoactivatable Trk receptors, designated optoTrks. Using one of the receptors, optoTrkB, we representatively show that $\operatorname{TrkB}$ signalling is rapidly and transiently controlled by a single photostimulation in a reversible, spatial and cell-specific manner. In addition, a prolonged patterned stimulation (illumination) of a neuronal cell line harbouring optoTrkB sustains ERK activity and induces neuronal differentiation. Finally, we further demonstrate the utility of optoTrk receptors by showing that blue-light exposure can trigger PI3K signalling activation and regulate neurite outgrowth, as well as filopodia formation in rat hippocampal neurons.

\section{Results}

Design of optoTrk receptors. As schematically illustrated in Supplementary Fig. 1a, we originally designed two different chimeric receptors containing full-length Trk, one consisting of Trk linked to PHR and the monomeric yellow fluorescent protein mCitrine (Trk-PHR-mCit), and the other consisting of Trk linked to $\mathrm{CIBN}$ and the monomeric cyan fluorescent protein mCerulean (Trk-CIBN-mCer). To test the PHR-CIBN interaction-mediated activation of Trk signalling in mammalian cells, we first constructed PHR- and CIBN-fused TrkB receptors and expressed a
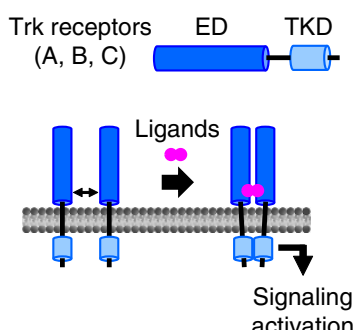

(ERK, AKT, $\mathrm{Ca}^{2+}$ ) b

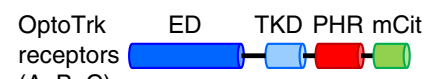
$(\mathrm{A}, \mathrm{B}, \mathrm{C})$

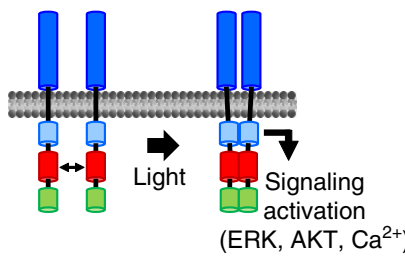

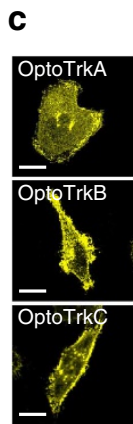

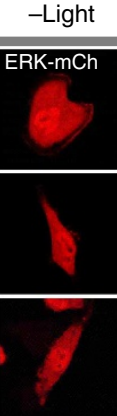
+ Light

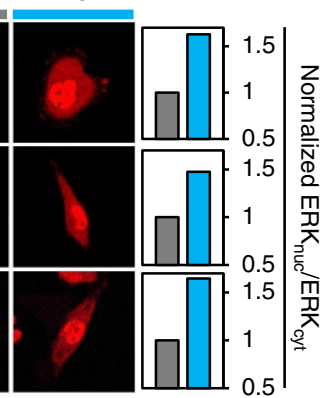

Figure 1 | Design and functional expression of photoactivatable Trk receptors. Schematic illustration of the molecular architecture and activation process of (a) Trk and (b) optoTrk receptors. Sites of induced monomer interaction are denoted by left right arrows. ED, extracellular domain; TKD, tyrosine kinase domain; and mCit, mCitrine. (c) Fluorescence images of optoTrk receptors and ERK-mCherry co-expressed in HeLa cells. ERK-mCherry (ERK-mCh) was imaged before and $5 \mathrm{~min}$ after a single-light stimulation ( $488 \mathrm{~nm}$, three 0.5 -s pulses, $6.5 \mu \mathrm{W}$ ). Graphs on the right show the normalized ratio of nucleus and cytosol ERK-mCh fluorescence $\left(\right.$ ERK $_{\text {nud }} / E^{2} K_{\text {cyt }}$ ) before (grey bar) and after (blue bar) blue-light exposure. Scale bars, $20 \mu m$. 
them together or individually in PC12 or HeLa cells, both of which do not express endogenous $\operatorname{TrkB}$ receptors ${ }^{13,14}$. Illumination of cotransfected PC12 cells using a blue LED array $(470 \mathrm{~nm}, 5.5 \mu \mathrm{W})$ caused an increase in endogenous phospho-ERK level (Supplementary Fig. 1b), suggesting a light-induced activation of ERK signalling. Unexpectedly, we found that TrkB-PHR-mCit alone was able to activate ERK in response to light stimulation, whereas TrkB-CIBN-mCer was not (Supplementary Fig. 1b). To further verify this phenomenon, we imaged intracellular $\mathrm{Ca}^{2+}$ level $\left(\left[\mathrm{Ca}^{2+}\right]_{\mathrm{i}}\right)$. Exposure of HeLa cells harbouring both of the two chimeric receptors to blue-light stimulation $(488 \mathrm{~nm}$, three $0.5 \mathrm{~s}$ pulses, $6.5 \mu \mathrm{W}$ ) evoked a $\mathrm{Ca}^{2}+$ transient, as demonstrated by an increase in the fluorescence intensity of cotransfected, intensiometric $\mathrm{Ca}^{2+}$ indicator $(\mathrm{R}-\mathrm{GECO} 1)^{15}$ shown in Supplementary Fig. 1c. Again, TrkB-PHR-mCit alone was sufficient to drive changes in $\left[\mathrm{Ca}^{2+}\right]_{i}$ in response to blue-light pulses, whereas TrkB-CIBN-mCit, mCitrine-tagged TrkB (TrkBmCit), or mCitrine-tagged PHR (PHR-mCit) did not elevate $\left[\mathrm{Ca}^{2+}\right]_{\mathrm{i}}$ after light stimulation (Supplementary Fig. 1c). Moreover, we did not observe light-triggered $\mathrm{Ca}^{2+}$ signal when TrkB receptor linked to a light-insensitive PHR mutant $\left(\mathrm{PHR}^{\mathrm{D} 387 \mathrm{~A}}\right)^{16}$ (TrkB-PHR $\left.{ }^{\mathrm{D} 387 \mathrm{~A}}-\mathrm{mCit}\right)$ was expressed (Supplementary Fig. 1c). Collectively, these results suggested that the PHR domain alone is able to confer a light-inducible interaction capacity on the linked TrkB receptor, thereby allowing it to mediate ERK and $\mathrm{Ca}^{2+}$ signalling in response to light. Thus, using a single light-dependent homo-interaction domain, we were able to construct a photoactivatable TrkB receptor, designated optoTrkB (Fig. 1b). Extending this concept to other members of the Trk family, we also constructed photoactivatable TrkA and TrkC receptors, optoTrkA and $\mathrm{C}$ (Fig. 1b).

Light-induced upregulation of canonical Trk signalling. To validate functional expression of optoTrks, we used biosensors of intracellular ERK, AKT and $\mathrm{Ca}^{2+}$ signalling activity to assay light-induced activation of canonical Trk signalling cascades in individual HeLa cells by live-cell imaging. Blue-light illumination of cells transfected with each optoTrk receptor clearly caused nuclear translocation of monomeric (m)Cherry-tagged ERK (ERK-mCh), indicating ERK pathway activation ${ }^{17}$ (Fig. 1c). In addition, light stimulation promoted plasma membrane localization of the pleckstrin homology domain of mCherrytagged AKT1 (mCh-PH $\mathrm{AKT}_{1}$ ) (Supplementary Fig. 2a), indicating $\mathrm{PI} 3 \mathrm{~K} / \mathrm{Akt}$ signalling activation ${ }^{18}$, and resulted in an increase in R-GECO1 signal, demonstrating $\left[\mathrm{Ca}^{2+}\right]_{\mathrm{i}}$ elevation (Supplementary Fig. 2b). Taken together, these results indicate that optoTrk receptors can induce upregulation of ERK, AKT and $\mathrm{Ca}^{2+}$ signalling pathways upon blue-light exposure. Among the modified receptors, we used optoTrkB for all follow-up studies.

Differential regulation of TrkB signalling by BDNF and light. TrkB signalling is activated by binding of TrkB receptor to its cognate ligand brain-derived neurotrophic factor $(\mathrm{BDNF})^{19}$, and the BDNF-binding sites within optoTrkB remain intact. Thus, to characterize the light-triggered activation of $\operatorname{TrkB}$ signalling cascades in more detail, we co-expressed optoTrkB with ERK-, PI3K- and $\mathrm{Ca}^{2+}$-biosensors, and monitored and analysed their responses to BDNF or blue-light stimulation in a time-dependent manner. At first, both stimuli caused an increase of ERK-mCh in the cell nucleus (Fig. 2a) and an accumulation of $\mathrm{mCh}^{-\mathrm{PH}_{\mathrm{AKT} 1}}$ at the plasma membrane (Fig. 2b). Kinetic measurements of ERK-mCh and $\mathrm{mCh}-\mathrm{PH}_{\mathrm{AKT} 1}$ translocation, however, revealed a common, stimulus-dependent difference: BDNF ( $50 \mathrm{ng} \mathrm{ml}^{-1}$ ) induced relatively slow, sustained signalling activation in optoTrkB-expressing cells during the examined periods
(Fig. 2a-c), whereas blue light triggered rapid, transient responses (Fig. 2a-c). For example, the normalized nucleus/ cytosol ratio of ERK-mCh was gradually increased and maintained at the elevated level, at least until $20 \mathrm{~min}$, in the presence of BDNF, but it was rapidly increased and declined to the baseline after a single light stimulation (Fig. 2c). Changes in ERK phosphorylation states, revealed by an immunoblot analysis, after applying the two stimuli were also consistent with this observation (Supplementary Fig. 3).

$\mathrm{Ca}^{2+}$ signalling kinetics showed a similar difference between the two stimuli. $\left[\mathrm{Ca}^{2+}\right]_{\mathrm{i}}$ elevation by $\operatorname{BDNF}\left(100 \mathrm{ng} \mathrm{ml}^{-1}\right)$ was induced slowly and sustained above the basal level for $>2 \mathrm{~min}$, whereas light-provoked $\mathrm{Ca}^{2}+$ transients was rapidly developed and disappeared within $90 \mathrm{~s}$ (Fig. 2d,e). The slow kinetics of TrkB signalling induced by activation with BDNF was attributable, in part, to the time required for BDNF diffusion and the concentration used. For example, doubling the amount of BDNF from 50 to $100 \mathrm{ng} \mathrm{ml}^{-1}$ shortened the time to the onset of a $\mathrm{Ca}^{2+}$ surge (defined as a $50 \%$ increase in $\left[\mathrm{Ca}^{2+}\right]_{\mathrm{i}}$ above baseline) from $67.9 \pm 7.6$ to as little as $27 \pm 3.3 \mathrm{~s}$ (mean \pm s.e.m.; Fig. 2e). Light stimulation caused a much faster $\mathrm{Ca}^{2+}$ surge (within $17.6 \pm 3.1 \mathrm{~s}$ (mean \pm s.e.m.); Fig. 2e), demonstrating the much greater temporal resolution of optically activated TrkB signalling.

In contrast to responses to BDNF, which were somewhat sustained, responses to light stimulation of optoTrkB were shortlived and rapidly recovered to baseline. This was true for ERK and AKT activity (Fig. $2 \mathrm{a}-\mathrm{c}$ ), as well as changes in $\left[\mathrm{Ca}^{2+}\right]_{\mathrm{i}}$ (Fig. $\left.2 \mathrm{~d}, \mathrm{e}\right)$. In addition, when we compared the optoTrkB performance by light with wild-type $\operatorname{TrkB}$ receptor activity by BDNF, similar differences in signalling activation kinetics were observed (Supplementary Fig. 4a-d). Taken together, these observations suggest that optoTrkB enables transient activation of the canonical TrkB signalling by a single photostimulation.

Signalling specificity of optoTrkB. We investigated the signalling specificity of optoTrkB. Light-induced ERK activation was totally eliminated by PD0325901, a MEK inhibitor (Supplementary Fig. 5a). In addition, LY294002, a PI3K inhibitor, and U73122, a PLC inhibitor, completely blocked light-mediated translocation of mCherry- $\mathrm{PH}_{\mathrm{AKT} 1}$ to the plasma membrane (Supplementary Fig. 5b) and light-triggered $\left[\mathrm{Ca}^{2+}\right]_{\mathrm{i}}$ elevation (Fig. 2d,e), respectively. Notably, those light-driven responses were abolished by K252a, a selective inhibitor of Trk tyrosine kinase activity (Supplementary Fig. 5 and Fig. 2d,e). These results indicate that the stimulatory effect of blue light on cells expressing optoTrkB is mediated through specific signalling cascades emanating from TrkB activation.

Versatile control of TrkB signalling using optoTrkB. We postulated that it would be feasible to achieve versatile control of light-gated TrkB signalling by manipulating illumination methods. To explore this, we took advantage of the fast response and recovery time ( $<90 \mathrm{~s}$ from activation to recovery; Fig. 2e) and simple readout of $\mathrm{Ca}^{2+}$ imaging. Exposure of HeLa cells cotransfected with optoTrkB and R-GECO1 to initial three 0.5-s blue-light pulses induced a rapid increase and subsequent decline in R-GECO1 fluorescence. After a 10-min interstimulus interval (ISI), a second photostimulation caused a change in intensity similar to that of the first stimulation (Fig. 3a,b and Supplementary Movie 1), demonstrating that this process can be controlled in a reversible manner. We further investigated the reversibility efficacy (defined as the ratio of the second peak amplitude to the first peak amplitude) of light-switched activation of TrkB-PLC $\gamma 1-\mathrm{Ca}^{2}+$ signalling by varying ISIs. At ISIs of or longer than $5 \mathrm{~min}$ ( 5 and $10 \mathrm{~min})$, a second $\mathrm{Ca}^{2+}$ transient was 
a

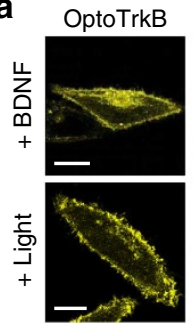

b

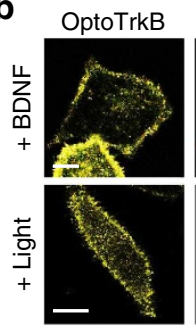

C
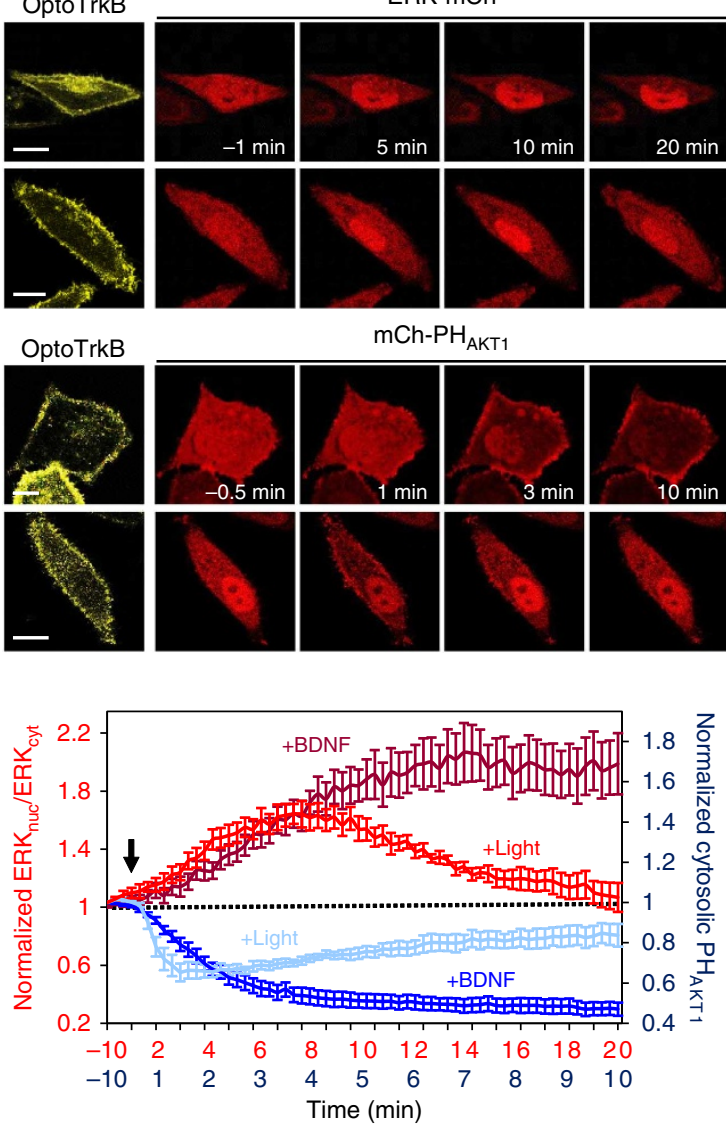

$\mathrm{mCh}-\mathrm{PH}_{\mathrm{AKT} 1}$
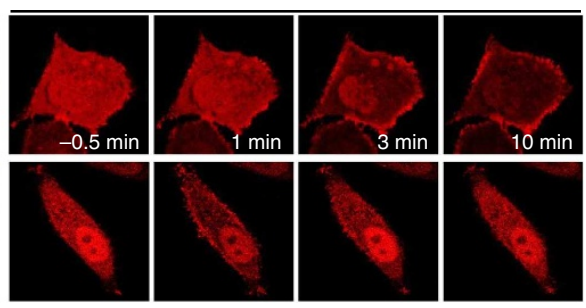

d
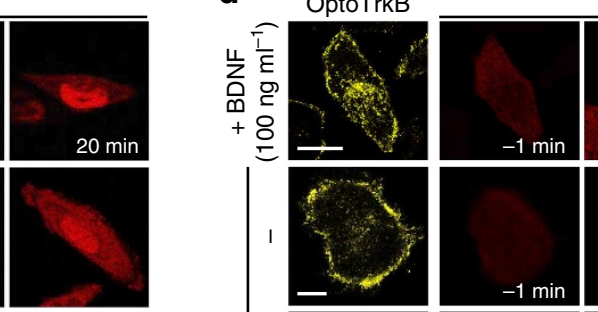

R-GECO1
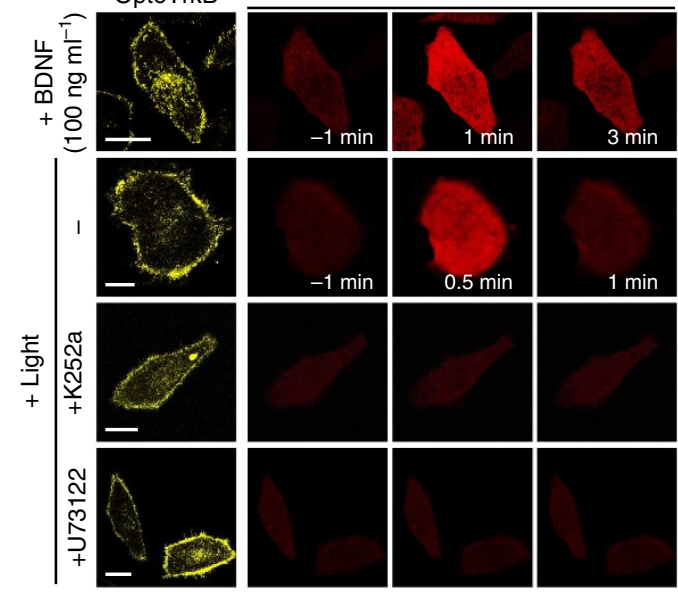

e

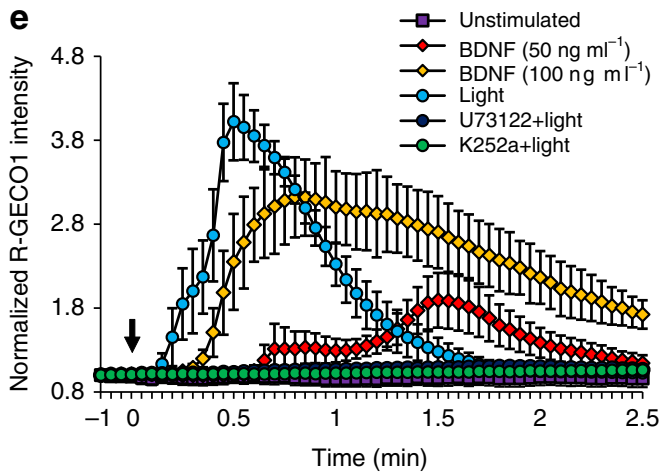

Figure 2 | BDNF and light stimulation differentially affected on TrkB signalling kinetics. Time-lapse images showing (a) nuclear translocation of

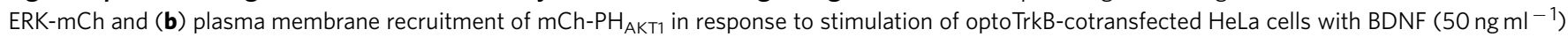
or blue light (three 0.5-s pulses, $6.5 \mu \mathrm{W}$ ). OptoTrkB expression is shown on the left. Scale bars, $20 \mu \mathrm{m}$. (c) Translocation kinetics of ERK-mCh and $\mathrm{mCh}-\mathrm{PH}_{\mathrm{AKT1}}$ after stimulation (arrow) of optoTrkB-cotransfected HeLa cells with BDNF or blue light. The graph shows quantified translocation by calculating the ratio of nuclear to cytosolic ERK-mCh $\left(E K_{\text {nud }} / E K_{\text {cyt }}\right.$ ) and changes in cytosolic $\mathrm{mCh}-\mathrm{PH}_{\mathrm{AKT1}}$, each normalized to baseline (at $-1 \mathrm{~min}$; dotted lines). Coloured numbers on each axis of the graph display different timescales and fold-change scales between $\mathrm{ERK}^{\mathrm{mCh}}$ (red) and $\mathrm{mCh}-\mathrm{PH} \mathrm{AKT}_{1}$ (blue) translocation. Error bars, \pm s.e.m. $(n>8)$. (d) Time course of changes in R-GECO1 fluorescence in HeLa cells expressing the indicated constructs before and after exposure to BDNF $\left(100 \mathrm{ng} \mathrm{ml}^{-1}\right)$ or light. Cells were photostimulated with or without a 30-min pre-incubation with $100 \mathrm{nM} \mathrm{K252a}$ or $2.5 \mu \mathrm{M}$ U73122. Scale bars, $20 \mu \mathrm{m}$. (e) $\mathrm{Ca}^{2+}$ signalling kinetics in HeLa transfected and stimulated as in d. Intracellular Ca ${ }^{2+}$ levels were quantified as changes in R-GECO1 fluorescence intensity divided by baseline fluorescence (at $-1 \mathrm{~min}$ ). Error bars, \pm s.e.m. ( $n>8$ ).

evoked with little or no loss of efficacy, whereas at ISIs shorter than $5 \mathrm{~min}$ (4 and $3 \mathrm{~min}$ ), a second stimulus elicited a reduced response in a stepwise fashion (Fig. $3 \mathrm{c}$ and Supplementary Fig. 6). And, delivery of two light stimuli spaced 2 min apart did not induce a second $\mathrm{Ca}^{2+}$ signal (Fig. 3c and Supplementary Fig. 6), overall suggesting a linear relationship between ISI and reversibility efficacy. Based on these results, we could repeatedly generate robust $\left[\mathrm{Ca}^{2+}\right]_{\mathrm{i}}$ elevations (at least five times) in a single cell with a train of blue-light pulses at a constant ISI (10 min) (Fig. 3d).

We next examined the subcellular resolution and spatial selectivity of optically activated TrkB signalling. Whole-cell illumination, in which light stimulation is confined to the whole area of a single cell, caused an increase in $\left[\mathrm{Ca}^{2+}\right]_{\mathrm{i}}$ simultaneously throughout the cell (Fig. 3e, top). In contrast, when blue-light pulses were delivered locally, $\mathrm{Ca}^{2+}$ signals were generated precisely from at each edge of a single cell and then propagated (Fig. 3e, middle and bottom). Thus, we were able to achieve fine spatial control of TrkB signalling. Moreover, in a mixed cell population containing HeLa cells with and without optoTrkB expression, global photostimulation, in which an illumination area covers more than one cell, selectively increased $\left[\mathrm{Ca}^{2+}\right]_{i}$ only in cells expressing optoTrkB (Fig. 3f and Supplementary Movie 2), demonstrating cell-specific modulation of signalling.

In addition to $\mathrm{Ca}^{2+}$ signal, an investigation of endogenous ERK activity demonstrated that TrkB signalling activity was light-controllable. Upon exposure of a population of optoTrkBtransfected HeLa cells to a 1-min global illumination $(470 \mathrm{~nm})$ using a blue LED array, phospho-ERK level was increased approximately fivefold after $5 \mathrm{~min}$ and declined after $25 \mathrm{~min}$ (Supplementary Fig. 7). A subsequent, second stimulation applied after a 25-min interval gave rise to a second increase in phosphoERK (Supplementary Fig. 7), confirming that endogenous signalling activity in a group of optoTrkB-expressing cells can be reversibly and synchronously modulated by light.

Light-induced neurite outgrowth by sustaining ERK activity. One of the prominent trophic effects of neurotrophins in Trkexpressing neuronal cells is promotion of neurite outgrowth. Nerve growth factor-induced neurite outgrowth in the rat pheochromocytoma cell line, PC12, has been extensively studied and characterized in this context ${ }^{20,21}$. Nerve growth factor treatment of PC12 cells, which endogenously express TrkA, 
a

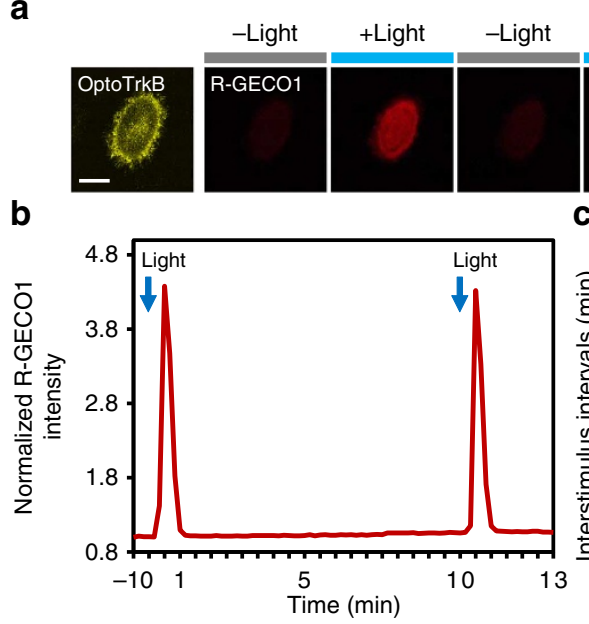

+Light -Light

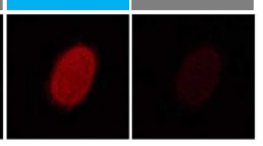

C Rev, efficacy (\%) 0255075100
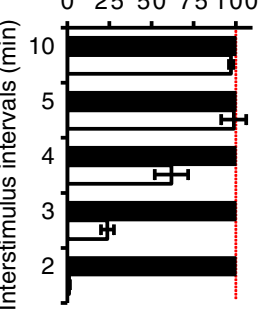
1st response
- 2nd response

d

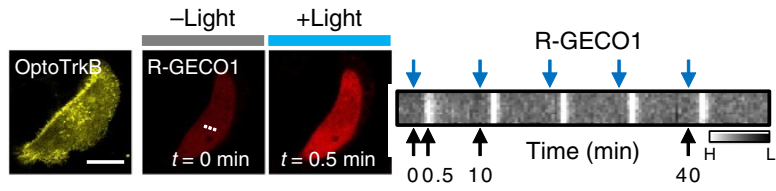

e OptoTrkB

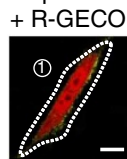

(2)
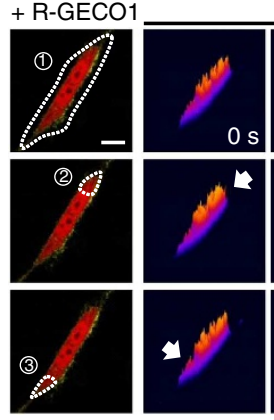

R-GECO1
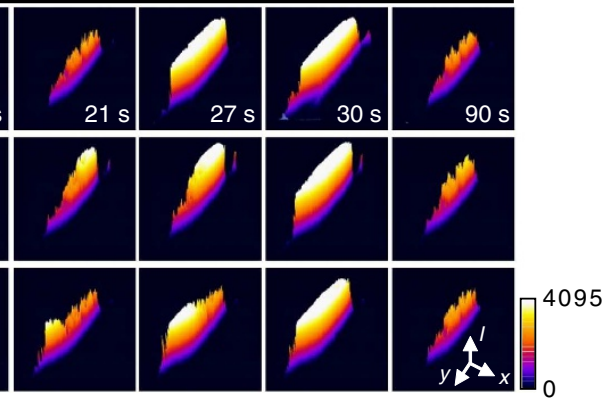

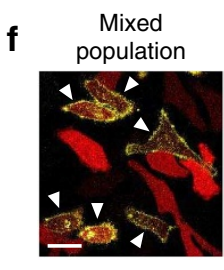

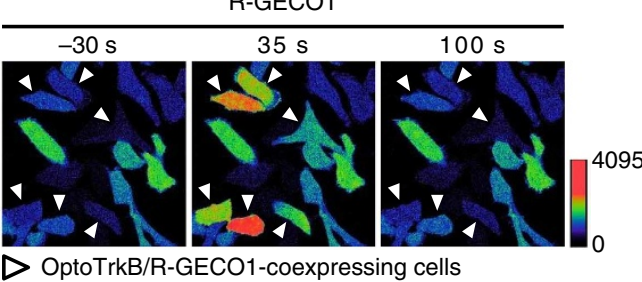

Figure 3 | Versatile modulation of TrkB signalling using light. (a) Fluorescence images of HeLa cells expressing the indicated constructs before and after delivery of three 0.5 -s pulses of blue light $(6.5 \mu \mathrm{W})$ twice with a 10-min interstimulus interval (ISI). Scale bar, $20 \mu \mathrm{m}$. (b) A normalized trajectory of changes in R-GECO1 fluorescence for the cell shown in a. Arrows indicate stimulation. (c) Relationship between reversibility (rev.) efficacy and ISI. Rev. efficacy was determined as the ratio of the normalized second peak R-GECO1 fluorescence (white bars) to the normalized first peak (black bars), expressed as a percentage, in each cotransfected HeLa cells stimulated twice with blue-light pulses with varying ISIs. Error bars, \pm s.e.m. ( $n>7$ ). (d) (Left) Fluorescence images of a HeLa cell expressing the indicated constructs before and $0.5 \mathrm{~min}$ after blue-light exposure. Scale bar, $20 \mu \mathrm{m}$. (Right) Kymograph drawn along the white dashed line within the cell shown on the left. The cell was illuminated five times (blue arrows above the kymograph) every 10 min. (e) (Left) A HeLa cell co-expressing optoTrkB and R-GECO1 was exposed to whole-cell (1) or local (2) and (3) light stimulation. The stimulated areas are outlined with white dashed lines. (Right) Time-lapse, pseudocolored surface intensity plots. Local stimulation is marked with arrows. Axis labelled ' I' represents intensity. Scale bar, $20 \mu \mathrm{m}$. (f) Selective induction of $\mathrm{Ca}^{2}+$ transients. HeLa cells transfected with R-GECO1 or cotransfected with optoTrkB and R-GECO1 (arrowheads) were co-cultured (mixed population) and simultaneously exposed to blue-light pulses. Time courses of R-GECO1 responses are represented by ratio images. Scale bar, $50 \mu \mathrm{m}$.

induces neuronal differentiation, a process in which sustained activation of ERK is essential ${ }^{20,21}$. Because TrkA and TrkB signalling pathways involve similar signalling proteins and PC12 cells expressing TrkB respond to BDNF by extending their neurites $^{22}$, we exploited this morphological response as a functional readout to evaluate whether light stimulation could mimic the biological effects of BDNF/TrkB signalling in optoTrkBtransfected PC12 cells. We first optimized illumination conditions by varying illumination duration and ISIs based on the lighttriggered ERK dynamics in HeLa (Supplementary Figs 3 and 4) at a constant laser power $(5.5 \mu \mathrm{W})$. Exposure of PC12 cells transfected with optoTrkB to a single 5-s illumination with blue LED $(470 \mathrm{~nm})$ led to a rapid increase in the level of active ERK within $5 \mathrm{~min}$, which diminished thereafter (Fig. 4a); these results paralleled those obtained in HeLa cells (Supplementary Figs 3 and 4). Notably, exposure to repetitive illumination ( $5 \mathrm{~s} \mathrm{on} / 5 \mathrm{~min}$ off) induced a high level of active ERK that was sustained for at least $4 \mathrm{~h}$ (Fig. 4a). Thus, we could produce long-duration ERK activity by stimulating optoTrkB-mediated signalling using a repetitive, alternating light-ON/light-OFF protocol.

Adopting this illumination protocol, we stimulated optoTrkBtransfected PC12 cells with blue-light pulses for $24 \mathrm{~h}$ and examined neurite outgrowth after phalloidin staining. In the absence of a stimulus, the morphology of undifferentiated PC12 cells was not affected by the expression of optoTrkB (Fig. 4b,c). In addition, repetitive illumination did not promote neurite formation in non-transfected PC12 cells (Fig. 4b, white arrowheads; and Fig. 4c, mock). However, 24-h repetitive irradiation markedly induced neurite extensions in optoTrkB-transfected
PC12 cells, producing an effect comparable to that of BDNF stimulation (Fig. 4b,c). The neurite outgrowth responses were affected by light intensity; the total neurite length and the length of the longest neurite was increased in an illumination dosedependent manner (Supplementary Fig. 8). In addition, we also monitored light-driven neurite outgrowth by using real-time, live-cell imaging (Supplementary Movie 3). In line with the staining data, repetitive light stimulation caused morphological changes in optoTrkB-expressing PC12 cells that did not occur in the absence of illumination. Taken together, these results indicate that BDNF/TrkB signalling activity was recapitulated by light control of optoTrkB. More importantly, they also suggest that optoTrkB expression, which, in theory, could lead to receptor auto-activation $^{23}$, did not spontaneously cause any marked biological consequences; expression of these receptors was functionally effective only when coupled with light stimulation.

Functional expression of optoTrkB in primary neurons. Finally, we examined optoTrkB performance in cultured rat hippocampal neurons. In a neuron at 7 DIV, which was cotransfected with optoTrkB and $\mathrm{mCh}-\mathrm{PH}_{\mathrm{AKT} 1}$, blue-light irradiation triggered rapid translocation of $\mathrm{mCh}-\mathrm{PH}_{\mathrm{AKT} 1}$ to the plasma membrane in the soma (Supplementary Fig. 9a-c), thereby demonstrating optoTrkB functionality. During this observation, we also found that filopodia- and lamellipodia-like structures (yellow arrows and blue arrowheads, respectively) were formed in the perisomatic region of the irradiated neuron (Supplementary Fig. 9d). Given that BDNF/TrkB signalling 
a

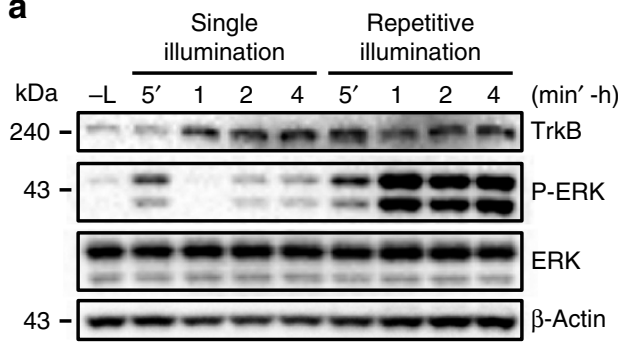

b

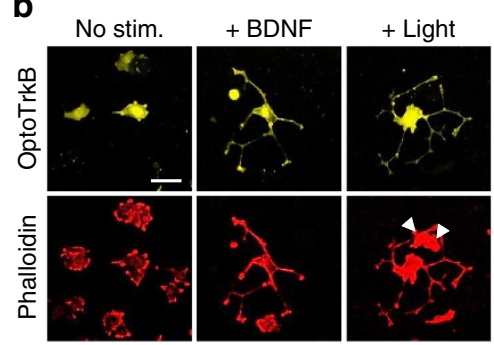

C

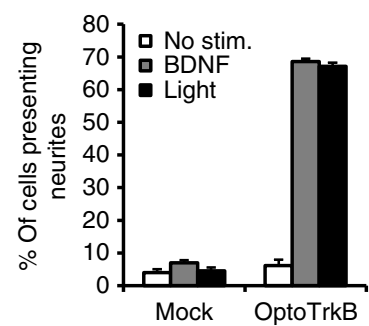

Figure 4 | Induction of neurite outgrowth by light-triggered, sustained ERK activity. (a) Sustained activation of ERK by light. Serum-starved (24h) optoTrkB-transfected PC12 cells were exposed to a single 5-s irradiation and left in the dark (single illumination) or to repetitive 5-s irradiations every $5 \mathrm{~min}$ (repetitive illumination) using a blue LED array $(470 \mathrm{~nm}$ and $5.5 \mu \mathrm{W})$. ERK activity was examined at the indicated times. $\beta$-Actin was included as a protein loading control. (b) Representative images of optoTrkB-transfected (upper panel) and phalloidin-stained (lower panel) PC12 cells. Serum-starved $\left(24 \mathrm{~h}\right.$ ) optoTrkB-transfected PC12 cells were unstimulated (no stim.) or stimulated with BDNF $\left(50 \mathrm{ng} \mathrm{ml}{ }^{-1}\right.$ ) or with a 5-s ON/5-min OFF illumination protocol using a blue LED array for an additional $24 \mathrm{~h}$. Phalloidin-stained cells marked with white arrowheads (on ' + Light' panel) denote non-transfected PC12 cells. Scale bar, $50 \mu \mathrm{m}$. (c) Quantification of neurite outgrowth. Non-transfected (mock) and optoTrkB-transfected PC12 (optoTrkB) cells treated as described in $\mathbf{b}$ were scored for the presence of neurites. Cells presenting neurites were defined as those with at least one neurite more than one cell-body diameter in length. Results are expressed as the percentage of neurite-bearing cells in the total number of counted cells. At least 100 cells were counted in each experiment and data are presented means \pm s.e. of three independent experiments.

activity has shown to drive an activity-dependent synapse formation process $^{24}$, in part by inducing filopodia and spine formation, and based on our observation, we next investigated light-induced filopodia formation using optoTrkB. For the visualization of the filopodial dynamics, we co-expressed mCherry-tagged LifeAct (mCh-LifeAct), which stains filamentous actin ${ }^{25}$. At first, live-cell imaging of hippocampal neurons overexpressing wild-type TrkB (TrkB-mCit) showed that BDNF $\left(100 \mathrm{ng} \mathrm{ml}^{-1}\right)$ treatment induced the filopodia formation (Supplementary Fig. 10a-c). The number of filopodia in neuritic processes was increased by nearly twofold after a 10-min administration of BDNF and plateaued thereafter (Supplementary Fig. 10c). Based on this, we next examined optoTrkB- and TrkB-mCit-transfected neurons after light and BDNF stimulation, respectively, for $30 \mathrm{~min}$. OptoTrkB expression per se did not augment neuronal filopodia density in the dark, comparable to that caused by TrkB-mCit expression in the absence of BDNF (Supplementary Fig. 11a-c). However, repetitive or continuous illumination induced a significant increase in the number of filopodia (Supplementary Fig. 11c). In addition, focal photostimulation elicited a spatially restricted formation of filopodia (Fig. 5a-c). Time-lapse imaging of a single neuron expressing optoTrkB and $\mathrm{mCh}$-LifeAct revealed that when exposed to a localized irradiation, the number of filopodia was increased only within the stimulated area but not in nearby non-stimulated area (Fig. 5b,c). Taken together, these results demonstrated an optical control of filopodial formation using optoTrkB in a temporally precise and spatially specific fashion.

We also tested the effects of pulsatile optoTrkB activation on the neurite outgrowth process in young hippocampal neurons. To this end, we expressed optoTrkB in freshly dissociated hippocampal neurons and stimulated them with BDNF $\left(100 \mathrm{ng} \mathrm{ml}^{-1}\right)$ or light $(25 \mu \mathrm{W})$ from DIV 1 to DIV 3 (for $48 \mathrm{~h}$ ), during which neuronal polarity is spontaneously established ${ }^{26}$ (control; Supplementary Fig. 12a). Blue-light illumination, as well as BDNF incubation, significantly increased the total neurite length (Supplementary Fig. 12b) and the number of branch points (Supplementary Fig. 12c) in young hippocampal neurons. BDNF incubation caused more prominent effects on neurite outgrowth than light stimulation, likely through the binding of BDNF to endogenous TrkB or p75 neurotrophic receptors ${ }^{19}$.

In addition to validating its functionality in primary neurons, we also monitored vesicular transport of optoTrkB receptor. Upon ligand binding at the axonal terminal, TrkB receptor is internalized and delivered in the retrograde direction, and thereby initiates retrograde signalling ${ }^{27}$. Therefore, BDNF signalling in neurons relies on proper transport of its TrkB receptor ${ }^{28}$. We compared the axonal transport of TrkB-mCit and optoTrkB in hippocampal neurons (Fig. 6a,b). A few vesicles were immobile (white asterisks) but most of them were mobile (arrows). And similarly as TrkB-mCit (Fig. 6a), optoTrkB receptors were actively retrogradely transported along the axon (Fig. 6b), demonstrating that the modified TrkB receptor was able to be internalized and transported in hippocampal neurons.

\section{Discussion}

Light control of cell signalling offers an unprecedented opportunity to interrogate the spatial and temporal dynamics of intracellular signalling circuits $^{29}$. Moreover, it also opens new avenues for investigating causal links between biochemical signalling and animal behaviour ${ }^{3}$. By simply combining a light-sensitive homointeraction module with a receptor-dimerization system-and without considerable engineering-we have added new singlecomponent optogenetic tools, optoTrk receptors (Fig. 1b,c and Supplementary Fig. 2), to the existing light-controllable toolkit. OptoTrk receptors provoke upregulation of canonical Trk pathways in response to blue-light exposure (Fig. 1c and Supplementary Fig. 2), demonstrating that receptor modification, the addition of PHR domain and mCitrine (Fig. 1b) does not alter signalling activity of native receptors. In addition, a photoactivatable form of TrkB mutated at Tyr-816 (Y816F) $)^{30}$ (TrkB ${ }^{\text {Y816F }}$-PHR-mCit), which is the PLC $\gamma 1$-binding site, failed to elevate $\left[\mathrm{Ca}^{2+}\right]_{i}$ after blue-light stimulation (Supplementary Fig. 13), further supporting that specific tyrosine phosphorylation, but not the C-terminally fused domains, influences the binding of proteins that are recruited at $\mathrm{TrkB}$ receptor.

The Trk receptors, like most RTKs, are activated by ligandinduced receptor dimerization. Accordingly, overexpression of them are prone to receptor auto-activation in the absence of stimulus $^{23}$. Indeed, for example, a modest induction of optoTrkB activation in the dark state, as demonstrated by an increase in ERK phosphorylation without illumination, was evident in HeLa cells (Supplementary Figs 3a and 5a), which may limit the utility of optoTrkB. However, we also observed that first, TrkB-mCit expression in HeLa cells was also shown to induce ERK phosphorylation in the absence of BDNF treatment (Supplementary Fig. 4c); and second, no significant increase in 

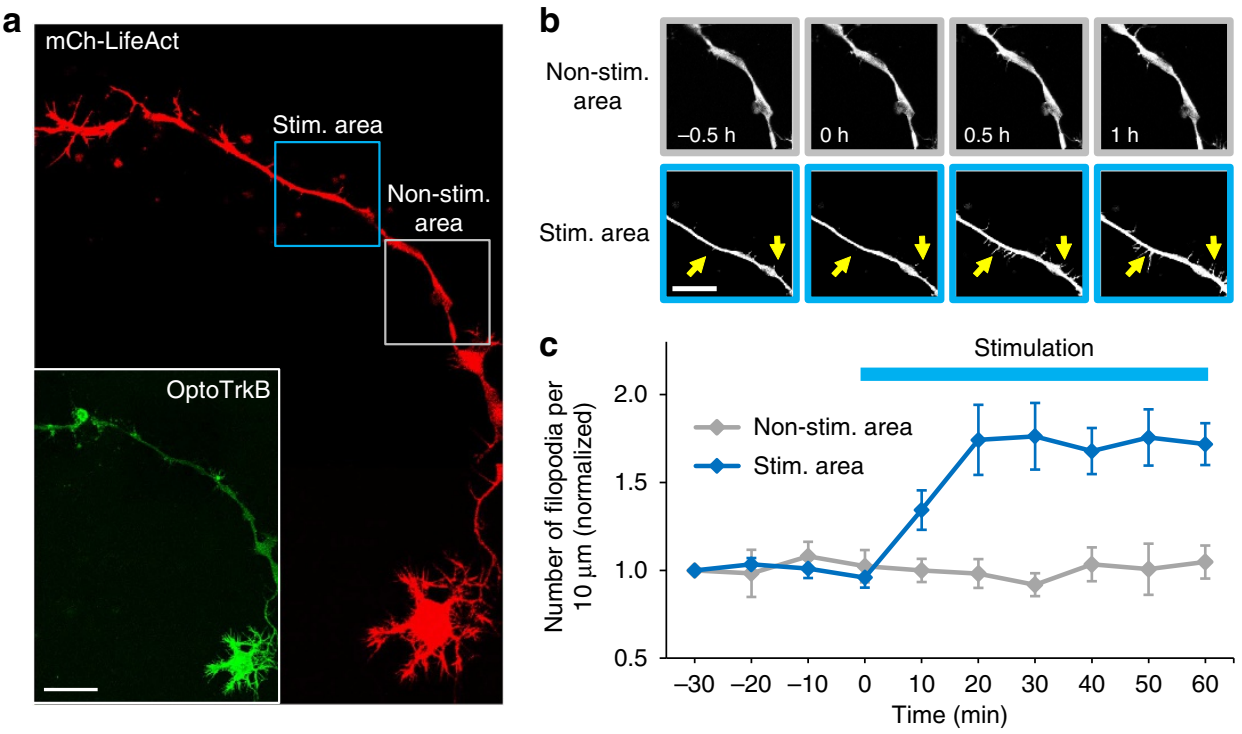

Figure 5 | Optical control of local filopodia formation in a hippocampal neuron expressing optoTrkB. (a) A hippocampal neuron (DIV 7) transfected with optoTrkB and $\mathrm{mCh}$-LifeAct. Scale bar, $50 \mu \mathrm{m}$. (b) Time-lapse images showing non-stimulated (non-stim., marked by grey box in a) and stimulated (stim., marked by blue box in $\mathbf{a}$ ) areas in the neuron shown in $\mathbf{a}$. The stimulated area was exposed to three 0.5-s pulses of blue light $(6.5 \mu \mathrm{W})$ every $30 \mathrm{~s}$ for $1 \mathrm{~h}$. Yellow arrows in the stimulated area highlight the newly formed filopodia. (c) Quantification of the number of filopodia. The number of filopodia in neuritic processes per $10 \mu \mathrm{m}$ was calculated at each time points and normalized to baseline (at -30 min). Error bars, \pm s.e.m. $(n=7)$. The blue bar in the graph indicates stimulation period.
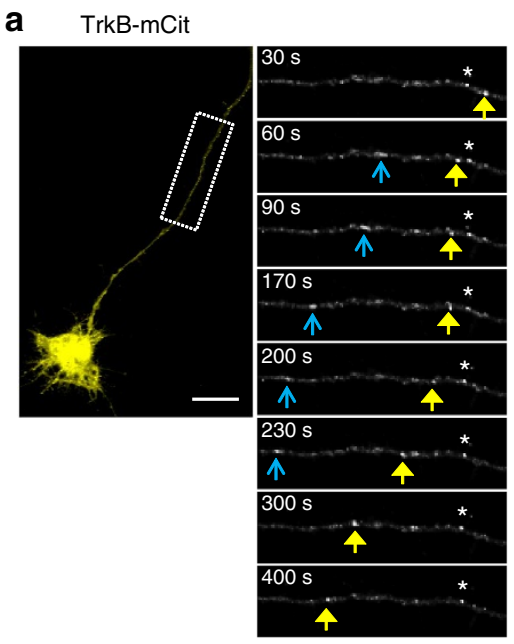

b

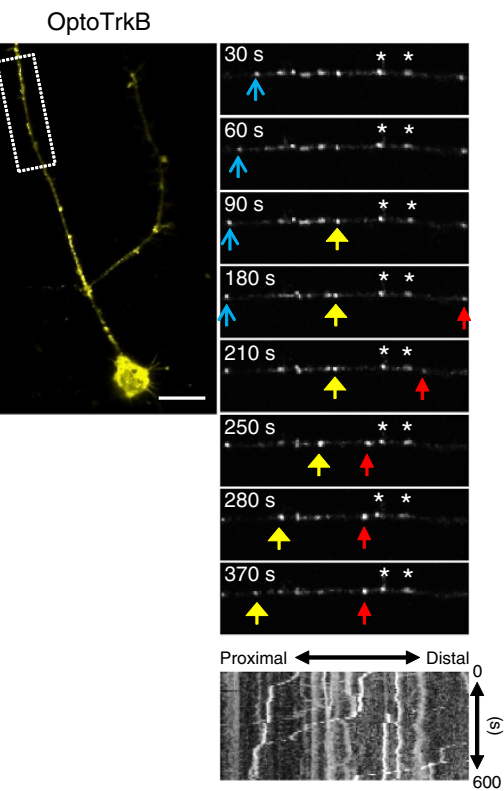

Figure 6 | Dynamic movements of wild-type TrkB and optoTrkB in axons. (a,b) Hippocampal neurons (DIV 8) were transfected with (a) TrkB-mCit or (b) optoTrkB, and movement of them were monitored along the axons (the longest neurite among others) by capturing images every $5 \mathrm{~s}$ for 10 min. The white boxes in representative TrkB-mCit and optoTrkB images indicate the area shown on right of each of them. The stationary and moving vesicles are marked by asterisks and arrows, respectively, and arrows with different colours indicate different individual vesicles. Kymographs show movement of TrkB-mCit and optoTrkB from a distal to proximal region of the axons, indicating retrograde transport. Scale bars, $20 \mu \mathrm{m}$.

background ERK activity was observed in PC12 cells regardless of optoTrkB expression level (Supplementary Fig. 14). Thus, these results suggest that not optoTrkB expression per se, but cell lines for its expression was responsible for the background signal activity.

A light-inducible protein-protein interaction with fast association and dissociation kinetics has the benefits of temporally precise control of cell signalling. Moreover, a genetically encoded dimerization system based on light-mediated protein homointeractions is highly favourable compared with that based on protein hetero-interactions. The photoactivatable receptors in this study, optoTrks, are based on PHR domain of cryptochrome 2 as a single light-responsive dimerizer. And upon blue-light exposure, optoTrk(B) rapidly (within seconds) and transiently (for a few minutes) activates corresponding signalling pathways (Fig. 2) without the need for exogenous cofactors, thus PHR meets the 
requirements for an ideal optogenetic dimerizer. The lightdependent homo-interaction property of PHR described here has been recently reported, showing that PHR can be oligomerized and form clusters by blue-light illumination in mammalian cells $^{31}$. Thus, as suggested ${ }^{31}$, our results further support that PHR can be harnessed as a single light-sensitive module to induce protein-protein interaction and can be adapted to a broad range of signalling molecules, including other RTK families.

Our light-dependent strategy offers several advantages over conventional ligand-based or pharmacological approaches, including those use organic compounds (such as CIDs) and engineered receptors ${ }^{9-1} \mathrm{P}$, for the control of receptor signalling. First, it has high temporal resolution, producing faster signalling responses (Fig. 2). Second, it is fully reversible in the minute timescale and enables precisely controlled repetitive activation (Fig. 3a-d). Third, it allows spatially restricted activation of cell signalling (Figs 3e and 5). Fourth, it lacks off-target effects and has cell-type specificity (Fig. 3f). In addition, it causes induction of transient and sustained protein activity by a single and a repetitive illumination, respectively (Supplementary Fig. 4 and Fig. 4a), thus providing a simple means to manipulate the functional duration of signalling.

In summary, optoTrkB enables versatile control of TrkB signalling and thus allows quantitative investigation of spatiotemporal signalling dynamics in vitro and in vivo. Moreover, it recapitulates $\mathrm{BDNF} / \mathrm{TrkB}$ signalling activity in response to light, thereby possessing beneficial properties from both cell biological and therapeutic perspectives ${ }^{19}$. Further engineering of optoTrk receptors to a truncated form to prevent endogenous ligand binding ${ }^{10}$ could make this tool readily applicable to in vivo systems.

\section{Methods}

Plasmid construction. TrkB cDNA was provided by K. Kaibuchi (Nagoya University, Nagoya), and TrkA, TrkB ${ }^{\mathrm{Y} 816 \mathrm{~F}}$ and TrkC $\mathrm{cDNAs}$ were provided by L.C. Schecterson (University of Washington, Seattle, WA). CIBN-pmGFP was provided by C.L. Tucker (University of Colorado Denver, Denver, CO). For efficient mammalian expression, the PHR-coding sequence ${ }^{7}$ was codon-optimized (here referred to as PHR) and listed in Supplementary Fig. 15. To construct chimeric TrkB, we first generated PHR-mCitrine by inserting NheI/AgeI-restricted PHR into the corresponding sites (NheI/AgeI) of pmCitrine-C1 (Clontech) and CIBNmCerulean by inserting NheI/AgeI-restricted CIBN from CIBN-pmGFP into the corresponding sites (NheI/AgeI) of pmCerulean-C1 (Clontech). Next, NheI/ BamHI-restricted TrkB, amplified by polymerase chain reaction (PCR) from TrkB cDNA using TrkB-F ( $5^{\prime}$-ggaaaagctagcATGTCGCCCTGGCCGAGGTGG-3 $\left.3^{\prime}\right)$ and TrkB-R (5'-ggaaaaggatccCCTAGGATGTCCAGGTAGACGG- $\left.3^{\prime}\right)$ primers was cloned into the corresponding sites (NheI/BamHI) in the multicloning site (MCS) of pEGFP-N1 (Clontech). The resulting TrkB-EGFP was further restricted with NheI/BsrGI and cloned into the corresponding sites (NheI/BsrGI) of pEGFP-C1 (Clontech) to yield TrkB-EGFP-MCS. Finally, $5^{\prime}$ blunt-ended and 3' MluIrestricted PHR-mCitrine, PCR-amplified from the PHR-mCitrine vector using PHR-F ( $5^{\prime}$-tcgccaccATGAAGATGGACAAAAA- $\left.3^{\prime}\right)$ and MluI-R $\left(5^{\prime}\right.$-gaaacgcgt TTACTTGTACAGCTCGTCCATGC- $3^{\prime}$ ) primers, or $5^{\prime}$ blunt-ended and $3^{\prime}$ MluI-restricted CIBN-mCerulean, PCR-amplified from CIBN-mCerulean vector using CIBN-F ( $5^{\prime}$-tcgccaccATGAATGGAGCTATAGGAGGTGA- $\left.3^{\prime}\right)$ and $M l u I-R$ primers, were inserted into TrkB-EGFP-MCS restricted with AgeI, filled in using Klenow fragment and further restricted with $M l u$ I. The resulting constructs, TrkB-PHR-mCitrine (optoTrkB) and TrkB-CIBN-mCerulean, encode full-length TrkB fused to PHR-mCitrine and CIBN-mCerulean, respectively, via a linker derived from pEGFP-N1 (DPPVAT). TrkB-CIBN-mCitrine was cloned following the same procedures described above, and TrkB-mCitrine was constructed by excising PHR from TrkB-PHR-mCitrine by using AgeI and re-ligating it. TrkB-PHR ${ }^{\mathrm{D} 387 \mathrm{~A}}$-mCitrine was generated by site-directed mutagenesis, as described $^{32}$, using AgeI-PHR-F (5' -ggatccaccggtcgccaccATGA-3 $^{\prime}$ ), (D387A)PHR-R ( $5^{\prime}$-CCAAGTCGGCGGCGAGAAGGGT $\overline{\left.A-3^{\prime}\right)}$, (D387A)PHR-F $\left(5^{\prime}\right.$-TACCCTTCT CGCCGCCGACTTGG- $\left.-3^{\prime}\right)$ and PHR-AgeI-R (5'-cggcagccaccggtCGCCA-3') primers. TrkB ${ }^{\mathrm{Y} 816 \mathrm{~F}_{-}}$-PHR-mCitrine was constructed by replacing TrkB from TrkBPHR-mCitrine with PCR-amplified TrkB ${ }^{\text {Y816F }}$ using BamHI and NheI restriction sites. TrkA-PHR-mCitrine (optoTrkA) and TrkC-PHR-mCitrine (optoTrkC) were constructed by inserting TrkA and TrkC cDNAs in place of the TrkB of optoTrkB using In-Fusion HD Cloning Kits (Clontech). The CMV (cytomegalovirus) promoter of optoTrkB was replaced by PGK (phosphoglycerate kinase 1) promoter of pPGK-Cre-bpA (plasmid 11543, Addgene) by AseI and NheI digestion.
ERK-mCherry was constructed by transferring the coding region of an Erk1 entry clone, created using Alliance for Cellular Signaling (AfCS) vector pEX EF1_Erk1 YFP (AfCS barcode: A05XP002A1NK), into an mCherry expression vector using LR Clonase (Invitrogen). mCherry- $\mathrm{PH}_{\mathrm{AKT1}}$ has been described previously ${ }^{18}$ and the R-GECO1 expression vector (CMV-R-GECO1) is available from Addgene (plasmid 32444). mCherry-LifeAct was constructed by replacing GFP from GFP-LifeAct ${ }^{25}$ to mCherry by using NheI and BsrGI restriction sites.

Reagents. Recombinant human BDNF was purchased from R\&D Systems (cat. no. 248-BD) and reconstituted in sterile phosphate-buffered saline (PBS). K252a (cat. no. K1639) and U73122 (PLC inhibitor; cat. no. U6756) were purchased from Sigma-Aldrich and dissolved in dimethyl sulfoxide (DMSO). LY294002 (PI3K inhibitor) was obtained from LC Laboratories (cat. no. L-7962) and dissolved in DMSO. PD0325901 (MEK inhibitor) was obtained from Cayman Chemical (cat. no. 13034) and dissolved in DMSO. Poly-D-lysine hydrobromide was obtained from Sigma-Aldrich (cat. no. P6407).

Cell culture and transfection. HeLa (American Type Culture Collection, ATCC) cells were cultured in Dulbecco's modified Eagle's medium (DMEM; cat. no. E15843; PAA Laboratories $\mathrm{GmbH}$ ) supplemented with $10 \%$ fetal bovine serum (FBS; cat. no. A12617; Gibco, Invitrogen) at $37^{\circ} \mathrm{C}$ in a humidified $10 \% \mathrm{CO}_{2}$ atmosphere The PC12 subline, Neuroscreen-1 (here referred to as PC12; Cellomics) ${ }^{33}$, was provided by T. Meyer (Stanford University, Stanford, CA) and cultured in F-12K media (cat. no. 10-025-CV; Cellgro) supplemented with $15 \%$ horse serum (cat. no. 16050-122; Gibco, Invitrogen) and $5 \%$ FBS at $37^{\circ} \mathrm{C}$ and $5 \% \mathrm{CO}_{2}$. Cells were transfected using a Microporator (Neon Transfection System; Invitrogen) according to the manufacturer's instructions. In brief, for live-cell imaging, $2 \times 10^{5}$ HeLa or PC12 cells were mixed with a total of $0.6 \mu \mathrm{g}$ DNA $(0.3 \mu \mathrm{g}$ each DNA in cotransfection experiments), electroporated, diluted 1:5 and seeded into a 96-well glass-bottom microplate (cat. no. MGB096-1-2-LG-L; Matrical Bioscience). For immunoblot analysis, $5 \times 10^{5} \mathrm{HeLa}$ or $2 \times 10^{6} \mathrm{PC} 12$ cells, transfected by electroporation with a total of $6 \mu \mathrm{g}$ DNA, were seeded per well of a six-well plate.

Preparation and transfection of hippocampal neurons. E18 pregnant SpragueDawley female rats were prepared for hippocampal cultures. All experimental procedures were approved by the Animal Ethic Committee at the Korea Advanced Institute of Science and Technology (KAIST; Daejeon, Korea). In brief, embryos were obtained from the rats and then placed into Hank's Balanced Salt Solution (HBSS) (cat. no. 14185-052; Gibco)-HEPES (10 mM; cat. no. 15630-080; Gibco) solution. Hippocampi were dissected from the embryos and incubated in $0.25 \%$ trypsin for $25 \mathrm{~min}$ at $37^{\circ} \mathrm{C}$ with tapping every $5 \mathrm{~min}$. Hippocampi were washed for three times with HBSS-HEPES, and triturated with a fire-polished Pasteur pipette. Tissues were dissociated after 10-20 trituration, and neurons were immediately plated in pre-equilibrated dishes or plates coated with $1 \mathrm{mg} \mathrm{ml}^{-1}$ poly-L-lysine (cat. no. P2636; Sigma) in plating medium. The plating medium consisted of Neurobasal medium (cat. no. 21103-049; Gibco) supplemented with $2 \%$ FBS, $2 \%$ B-27 (cat. no. 17504-044; Gibco), 2\% glutamax (cat. no. 35050-061; Gibco) and 2\% penicillin-streptomycin (cat. no. 15140-122; Gibco). The neurons were incubated at $37^{\circ} \mathrm{C}$ in a humidified $5 \% \mathrm{CO}_{2}$ atmosphere. Plating medium were replaced with maintaining medium (plating medium without FBS) within 6-24h. Neurons were transfected with LipofectamineLTX and plus reagent (Invitrogen) in Opti-MEM (Invitrogen) following manufacturer's instruction. In brief, total $0.5 \mu \mathrm{g}$ of DNA was incubated with $0.5 \mu \mathrm{l}$ of plus reagent in $100 \mu \mathrm{l}$ of Opti-MEM for $5 \mathrm{~min}$, and then $0.5 \mu \mathrm{l}$ LipofectamineLTX was added to the mixture. After $20 \mathrm{~min}$ incubation at room temperature, $20 \mu \mathrm{l}$ from $100 \mu \mathrm{l}$ mixture was dropped into a well of a 96-well microplate where the medium was completely replaced to $100 \mu \mathrm{l}$ of the new maintaining medium. The medium was then replaced to the kept conditioned medium after $45 \mathrm{~min}$.

Live-cell imaging, photostimulation and data acquisition. Cells were imaged $24 \mathrm{~h}$ after transfection. Before monitoring ERK and AKT activity, transfected HeLa cells were serum-starved for $6 \mathrm{~h}$. Just before imaging ERK, AKT and $\mathrm{Ca}^{2+}$ activity, the medium was replaced with Dulbecco's PBS containing glucose (cat. no. 14287080; Invitrogen). Neurite outgrowth was observed by imaging transfected PC12 cells in serum-free medium (F-12K).

Live-cell imaging was performed using a Nikon A1R confocal microscope (Nikon Instruments) mounted onto a Nikon Eclipse Ti body and equipped with CFI Plan Apochromat VC objectives $(60 \times / 1$.4-numerical aperture $(\mathrm{NA})$ oil or $40 \times / 0.95$-NA air; Nikon) and a Chamlide TC system (maintained $37^{\circ} \mathrm{C}$ and 10 or $5 \% \mathrm{CO}_{2}$; Live Cell Instrument, Inc., Korea). mCerulean, GFP, mCitrine and mCherry images $\left(512 \times 512\right.$ pixels, $\left.72.7 \mu \mathrm{m}^{2}\right)$ were taken using $457-, 488-, 514-$ and 561-nm laser lines, respectively. A built-in photo activation illumination unit was used to excite the illumination volume (generally confined to a single cell except for the experiments shown in Fig. 3f and Supplementary Movies 2 and 3) with 488-nm light during live-cell imaging. The 488-nm laser intensity used for photostimulation $\left(6.5 \mu \mathrm{W}\right.$ per $72.7 \mathrm{~m}^{2}=1.27 \mathrm{~mW} \mathrm{~cm}^{-2}$; here denoted simply as $6.5 \mu \mathrm{W})$ was measured right above the objective. The images were analysed with Nikon imaging software (NIS-element AR 64-bit version 3.10; Laboratory Imaging) and MetaMorph software (version 7.6.0.0; MDS Analytical Technologies). 
Electronics. An optical power meter (cat. no. 8230E; ADCMT, Japan) was used to measure blue-light intensity. We used two types of illumination devices consisting of 24 and 96 blue LEDs (cat. no. PP465-8L63-ESESBI; Photron, Korea) mounted on a customized array (Live Cell Instrument, Inc.; Supplementary Fig. 16). For the 24 LED array, each set of four LEDs was positioned at the centre of each well of a six-well plate. The LEDs emitted an equal intensity blue light onto cells in a six-well plate $(5.5 \mu \mathrm{W}$, measured $1 \mathrm{~cm}$ from the LEDs) and the light pulses were controlled using a digital time switch (cat. no. TR 611 top2; Theben). The 96 LEDs were positioned to fit into wells of a 96-well glass-bottom microplate, and eight LEDs in each column were simultaneously controlled using Micro-manager software (version 1.4.15) to allow independent control of 12 columns.

Immunoblot analysis. Whole-cell lysates were prepared in the dark using PROPREP solution (cat. no. 17081; iNtRON Biotechnology) and $20 \mu \mathrm{g}$ of total protein was resolved on a NuPAGE Novex 4-12\% Bis-Tris gel (Invitrogen). Proteins were transferred to a nitrocellulose membrane using an iBlot Transfer Stack and iBlot Gel Transfer Device (Invitrogen) according to the manufacturer's instructions. The membrane was immunoblotted by incubating first with primary rabbit anti-TrkB (1:1,000; cat. no. 4606; Cell Signaling Technology), rabbit anti-phospho-Erk1/2 (1:1,000; cat. no. 9101; Cell Signaling Technology), rabbit anti-Erk1/2 (1:1,000; cat. no. 9102; Cell Signaling Technology), rabbit anti- $\beta$-tubulin (1:1,000; cat. no. 04-1049; Millipore) and/or mouse anti- $\beta$-actin (1:1,000; cat. no. A5316; Sigma) antibodies, and then with horseradish peroxidase conjugated anti-rabbit immunoglobulin (IgG-HRP) (1:3,000; cat. no. NA934V; GE Healthcare) or anti-mouse IgG-HRP (1:3,000; cat. no. sc-2005; Santa Cruz Biotechnology) secondary antibodies, as appropriate, using standard procedures. Proteins were visualized using SuperSignal West Pico Chemiluminescent Substrate (cat. no. 34080; Thermo Scientific) and a ChemiDoc MP system (Bio-Rad). For the blots shown in Supplementary Figs 4 and 7, following secondary antibodies were used: goat-antimouse IR Dye 800CW (1:3,000; cat. no. 926-32210; LI-COR) or goat-anti-rabbit IR Dye 600CW (1:3,000; cat. no. 626-68071; LI-COR). These blots were scanned by Odessey CLx Infrared Imaging system (LI-COR). Densitometric analysis was carried out on the immunoblots using MetaMorph software. Uncropped gel scans of all western blots in this paper were presented in Supplementary Fig. 17.

Neurite outgrowth assay. OptoTrkB-transfected PC12 cells in poly-D-lysinecoated 96-well plates were serum-starved for $24 \mathrm{~h}$ and either left unstimulated, treated with BDNF (50 $\mathrm{ng} \mathrm{ml}^{-1}$ ) or exposed to pulsatile illumination (5-s irradiation every $5 \mathrm{~min}, 470 \mathrm{~nm}, 5.5 \mu \mathrm{W}$ ) administered with a blue LED array for an additional $24 \mathrm{~h}$ in serum-free media at $37^{\circ} \mathrm{C}$ in a humidified $5 \% \mathrm{CO}_{2}$ environment. All the samples were kept in the dark for the duration. Cells were fixed by incubating with a $4 \%$ methanol-free formaldehyde solution in PBS (cat. no. 28908; Thermo Scientific) and permeabilized with $0.1 \%$ Triton X-100 in PBS. Filamentous actin was stained with Alexa Fluor 594-conjugated phalloidin (cat. no. A12381; Invitrogen) and cells were imaged using a confocal microscope. The number of neurite-presenting cells, the total neurite length and the length of the longest neurite were measured and analysed with ImageJ software (http:// rsbweb.nih.gov/ij/).

Freshly dissociated hippocampal neurons (DIV 0) were transfected with optoTrkB and after $24 \mathrm{~h}$, they were either non-stimulated or stimulated with BDNF (100 $\mathrm{ng} \mathrm{ml}{ }^{-1}$ ) or light pulses $(25 \mu \mathrm{W})$ at $37^{\circ} \mathrm{C}$ in a humidified $5 \% \mathrm{CO}_{2}$ environment for additional $48 \mathrm{~h}$. All the samples were kept in the dark for the duration. Cells were fixed by incubating with a $4 \%$ methanol-free formaldehyde solution in PBS and imaged using a confocal microscope. The total neurite length and the number of branch points per cell were measured and analysed using MetaMorph software.

Filopodia formation assay. For the live-cell imaging, neurons at DIV 5-7 were transfected with TrkB-mCitrine or optoTrkB and mCherry-LifeAct. After $24 \mathrm{~h}$, they were treated with BDNF $\left(100 \mathrm{ng} \mathrm{ml}^{-1}\right)$ or light pulses $(6.5 \mu \mathrm{W})$ for $1 \mathrm{~h}$ and images were taken using a confocal microscope. For the analysis of fixed samples, neurons at DIV 5 were transfected with TrkB-mCitrine or optoTrkB and exposed to $\operatorname{BDNF}\left(100 \mathrm{ng} \mathrm{ml}^{-1}\right)$ or light pulses using a blue LED array $(25 \mu \mathrm{W})$ for $30 \mathrm{~min}$, respectively. Cells were fixed, permeabilized, stained with Alexa Fluor 594-conjugated phalloidin, and then imaged using a confocal microscope. Filopodia were defined as any protrusion under $10 \mu \mathrm{m}$ in length. The number of filopodia per $10 \mu \mathrm{m}$ were measured and analysed using Nikon imaging software.

\section{References}

1. Rosenbaum, D. M., Rasmussen, S. G. F. \& Kobilka, B. K. The structure and function of G-protein-coupled receptors. Nature 459, 356-363 (2009).

2. Lemmon, M. A. \& Schlessinger, J. Cell signaling by receptor tyrosine kinases. Cell 141, 1117-1134 (2010).

3. Airan, R. D., Thompson, K. R., Fenno, L. E., Bernstein, H. \& Deisseroth, K. Temporally precise in vivo control of intracellular signalling. Nature 458, 1025-1029 (2009).
4. Oh, E., Maejima, T., Liu, C., Deneris, E. \& Herlitze, S. Substitution of 5-HT $\mathrm{H}_{1 \mathrm{~A}}$ receptor signaling by a light-activated $\mathrm{G}$ protein-coupled receptor. J. Biol. Chem. 285, 30825-30836 (2010).

5. Yazawa, M., Sadaghiani, A. M., Hsueh, B. \& Dolmetsch, R. E. Induction of protein-protein interactions in live cells using light. Nat. Biotechnol. 27, 941-U105 (2009).

6. Levskaya, A., Weiner, O. D., Lim, W. A. \& Voigt, C. A. Spatiotemporal control of cell signalling using a light-switchable protein interaction. Nature 461, 997-1001 (2009).

7. Kennedy, M. J. et al. Rapid blue-light-mediated induction of protein interactions in living cells. Nat. Methods 7, 973-U948 (2010).

8. Rutkowska, A. \& Schultz, C. Protein tango: The toolbox to capture interacting partners. Angew. Chem. Int. Ed. Engl. 51, 8166-8176 (2012).

9. Muthuswamy, S. K., Gilman, M. \& Brugge, J. S. Controlled dimerization of ErbB receptors provides evidence for differential signaling by homo- and heterodimers. Mol. Cell. Biol. 19, 6845-6857 (1999).

10. Whitney, M. L., Otto, K. G., Blau, C. A., Reinecke, H. \& Murry, C. E. Control of myoblast proliferation with a synthetic ligand. J. Biol. Chem. 276, 41191-41196 (2001).

11. Alfa, R. W., Tuszynski, M. H. \& Blesch, A. A novel inducible tyrosine kinase receptor to regulate signal transduction and neurite outgrowth. J. Neurosci. Res. 87, 2624-2631 (2009).

12. Patapoutin, A. \& Reichardt, L. F. Trk receptors: mediators of neurotrophin action. Curr. Opin. Neurobiol. 11, 272-280 (2001).

13. Squinto, S. P. et al. TrkB encodes a functional receptor for brain-derived neurotrophic factor and neurotrophin-3 but not nerve growth-factor. Cell 65, 885-893 (1991).

14. Watson, F. L. et al. Rapid nuclear responses to target-derived neurotrophins require retrograde transport of ligand-receptor complex. J. Neurosci. 19, 7889-7900 (1999).

15. Zhao, Y. X. et al. An expanded palette of genetically encoded $\mathrm{Ca}^{2+}$ indicators. Science 333, 1888-1891 (2011).

16. Liu, H. T. et al. Photoexcited CRY2 interacts with CIB1 to regulate transcription and floral initiation in Arabidopsis. Science 322, 1535-1539 (2008).

17. Shankaran, H. et al. Rapid and sustained nuclear-cytoplasmic ERK oscillations induced by epidermal growth factor. Mol. Syst. Biol. 5, 332 (2009).

18. Yang, H. W. et al. Cooperative activation of PI3K by Ras and Rho family small GTPases. Mol. Cell 47, 281-290 (2012).

19. Nagahara, A. H. \& Tuszynski, M. H. Potential therapeutic uses of BDNF in neurological and psychiatric disorders. Nat. Rev. Drug Discov. 10, 209-219 (2011).

20. Marshall, C. J. Specificity of receptor tyrosine kinase signaling - transient versus sustained extracellular signal-regulated kinase activation. Cell 80, 179-185 (1995).

21. Vaudry, D., Stork, P. J. S., Lazarovici, P. \& Eiden, L. E. Signaling pathways for PC12 cell differentiation: making the right connections. Science 296, 1648-1649 (2002).

22. Iwasaki, Y., Ishikawa, M., Okada, N. \& Koizumi, S. Induction of a distinct morphology and signal transduction in TrkB/PC12 cells by nerve growth factor and brain-derived neurotrophic factor. J. Neurochem. 68, 927-934 (1997).

23. Schecterson, L. C. \& Bothwell, M. Neurotrophin receptors: old friends with new partners. Dev. Neurobiol. 70, 332-338 (2010).

24. Lu, B. BDNF and activity-dependent synaptic modulation. Learn. Mem. 10, 86-98 (2003).

25. Riedl, J. et al. Lifeact: a versatile marker to visualize F-actin. Nat. Methods 5, 605-607 (2008).

26. Fukata, Y., Kimura, T. \& Kaibuchi, K. Axon specification in hippocampal neurons. Neurosci. Res. 43, 305-315 (2002).

27. Segal, R. A. Selectivity in neurotrophin signaling: theme and variations. Ann. Rev. Neurosci. 26, 299-330 (2003).

28. Huang, E. J. \& Reichardt, L. F. Trk receptors: roles in neuronal signal transduction. Ann. Rev. Biochem. 72, 609-642 (2003).

29. Toettcher, J. E., Voigt, C. A., Weiner, O. D. \& Lim, W. A. The promise of optogenetics in cell biology: interrogating molecular circuits in space and time. Nat. Methods 8, 35-38 (2011).

30. Hollis, E. R., Jamshidi, P., Low, K., Blesch, A. \& Tuszynski, M. H. Induction of corticospinal regeneration by lentiviral trkB-induced Erk activation. Proc. Natl Acad. Sci. USA 106, 7215-7220 (2009).

31. Bugaj, L. J., Choksi, A. T., Mesuda, C. K., Kane, R. S. \& Schaffer, D. V. Optogenetic protein clustering and signaling activation in mammalian cells Nat. Methods 10, 249-252 (2013).

32. Heckman, K. L. \& Pease, L. R. Gene splicing and mutagenesis by PCR-driven overlap extension. Nat. Protoc. 2, 924-932 (2007).

33. Dijkmans, T. F. et al. Temporal and functional dynamics of the transcriptome during nerve growth factor-induced differentiation. J. Neurochem. 105, 2388-2403 (2008). 


\section{Acknowledgements}

We thank K. Kaibuchi, L. C. Schecterson, and C. L. Tucker for sharing plasmids. In addition, we thank Su Yeon Choi from Eunjoon Kim's laboratory at IBS/KAIST and Seock Kang from Myoung-Goo Kang's laboratory at IBS for guiding us how to culture rat primary hippocampal neurons. This work was supported by the National Research Foundation of Korea Stem Cell Program (no. 2011-0019509), the Intelligent Synthetic Biology Center of Global Frontier Project (no. 2011-0031955), the National Leading Research Laboratory Program (to D.K.; 2011-0028772), the National Research Foundation of Korea (NRF) grant funded by the Ministry of Science and Future Planning (2010-0027941) and the Korea Advanced Institute of Science and Technology Future Systems Healthcare Project funded by the Ministry of Science, Information and Communication Technology \& Future Planning in Korea.

\section{Author contributions}

W.D.H. and K.-Y.C. conceived the idea, and W.D.H. directed the work; K.-Y.C. and

D.W. designed experiments; K.-Y.C., D.W., H.J., S.K., J.W., T.K. and H.P. performed experiments; S.L., N.K. and H.W.Y. supervised microscopy experiments and assisted data analysis; J.-Y.P., E.M.H. and D.K. gave specific comments on overall experiments and data configuration; K.-Y.C., D.W., H.J. and W.D.H. wrote the paper.

\section{Additional information}

Supplementary Information accompanies this paper at http://www.nature.com/ naturecommunications

Competing financial interests: The authors declare no competing financial interests.

Reprints and permission information is available online at http://npg.nature.com/ reprintsandpermissions/

How to cite this article: Chang, K.-Y. et al. Light-inducible receptor tyrosine kinases that regulate neurotrophin signalling. Nat. Commun. 5:4057 doi: 10.1038/ncomms5057 (2014) 